\title{
Estudio Transversal de Procesos Administrativos en Municipios de la Huasteca Norte
}

\author{
Mtro. Pablo Martínez González. \\ UASLP, Unidad Académica Multidisciplinaria, Zona Huasteca, México \\ Dr. Hugo Jesús Ochoa Hernández \\ UASLP, Facultad de Economía, México \\ Dr. Mario Eduardo Ibarra Cortés
}

UASLP, Facultad de Contaduría y Administración, México

Doi:10.19044/esj.2018.v14n26p232 URL:http://dx.doi.org/10.19044/esj.2018.v14n26p232

\begin{abstract}
This paper focuses, first, on presenting a tool designed as a methodological proposal to help the municipal administrations to improve their administrative processes. This is possible by taking into consideration the phases that integrates it (Planning, Organization, Integration, Direction and Control) in order to identify the steps of the process where there are failures. This, however, makes it possible to detect them, apply corrective measures, and ensure that they are able to carry out processes of continuous improvement. Secondly, the document presents a study in which a methodology was applied. Here, it could be documented that for the case of the municipalities of Tamasopo, Tamuín and Ciudad Valles in the State of San Luis Potosí, a lack of systematization was detected in the administrative processes. The results have allowed us to perceive a differentiated opinion between the perception that the Heads of Department have regarding the application of the Administrative Process and the opinion that operative workers have regarding the phases of planning, organization, integration, direction, and control.
\end{abstract}

Keywords: Administration, Administrative Process, Systems, Planning, Organization, Management, Integration, Control

Resumen

El presente trabajo expone en primer término una herramienta diseñada como propuesta metodológica para ayudar a las administraciones municipales a mejorar sus procesos administrativos, tomando en consideración las fases que lo integran (Planeación, Organización, Integración, Dirección y Control), esto con el fin de identificar los pasos del 
proceso en los cuales se están produciendo fallas para que así estas puedan ser detectadas, se puedan aplicar medidas correctivas al respecto y sea luego posible llevar a cabo procesos de mejora continua. En segundo término, el documento presenta un estudio en donde se aplicó una metodología a partir de la cual se pudo documentar que, en el caso de los municipios de Tamasopo, Tamuín y Ciudad Valles en el Estado de San Luis Potosí, se detecta una falta de sistematización en los procesos administrativos. Los resultados nos han permitido percibir una opinión diferenciada entre la percepción que tienen los Jefes de Departamento en cuanto a la aplicación del Proceso Administrativo y la opinión que tienen los trabajadores operativos con respecto a las fases de planeación, organización, integración, dirección y control.

Palabras Clave: Administración, Proceso Administrativo, Sistemas, Planeación, Organización, Dirección, Integración, Control.

\section{Introducción}

Hoy en día es necesario recordar que, a lo largo de su prolongada existencia, los gobiernos han sido objeto de muchas reorganizaciones y reformas. Estas reformas se han dado principalmente desde la perspectiva de la administración pública, y como es mencionado por Peters (2005) "el motivo primordial de dichas reformas ha sido el incrementar la eficiencia del sector público así como la aparente incapacidad de esos gobiernos para manejarse a sí mismos tan bien como lo hacen otras organizaciones de la sociedad" (p.1).

Merino (2004) señala que "la agenda de la investigación en México se ha enfocado en dos polos opuestos; por una parte se ubican los estudios que han tendido a detectar y discutir las dificultades tipo macro, orientados principalmente hacia las reformas municipales de orden normativo, y por otro lado se encuentran los trabajos que han intentado superar esa visión tipo macro para ir en busca de las prácticas de gestión pública que, a pesar de todo, han logrado resolver problemas concretos de administración pública a partir de la introducción de pequeñas pero exitosas innovaciones en los gobiernos locales" (p.15).

Hoy en día a la problemática de la administración pública, si bien se enfoca en problemas de tipo financiero-administrativo, con la globalización y la firma de tratados internacionales "se han sumado otras temáticas tales como los derechos electrónicos, el gobierno electrónico y la transparencia y rendición de cuentas ante una sociedad cada día más interesada en los temas de la administración pública" (Mballa, 2018).

Aunado a lo anterior, el tema de la administración pública y la eficiencia en sus procesos representa un área de estudio fundamental para el conocimiento de las decisiones tomadas en los organismos públicos, dado que día a día las decisiones son tomadas en diferentes ámbitos de la administración 
pública; tal y como lo señala Castellanos (2014), “en el ámbito del gobierno y la administración pública es muy fácil identificar tres procesos de tomas de decisiones: en el ámbito legislativo, en el ámbito jurisdiccional judicial, y desde luego, en el proceso administrativo público" (p.72).

Por otra parte, para Martínez (2014) uno de los problemas más importantes en la actualidad tiene que ver con la eficiencia del gasto público. A ese respecto señala que "hay una desigualdad entre municipios que puede ocasionar condiciones para el desarrollo en algunas zonas en detrimento de otras zonas. Sin embargo, se presenta evidencia de que las acciones que los gobiernos municipales pueden hacer utilizando los escasos recursos de los fondos del ramo 33 para atender las mínimas responsabilidades que se les encomienda en el artículo 115 constitucional pueden impactar de forma muy significativa en la calidad de vida de los habitantes" (p.103).

Continúa señalando Martínez (2014) que "en este orden de ideas quedan abiertas las preguntas ¿Por qué los municipios parecen concentrar los recursos en sus cabeceras municipales? ¿Qué condiciones necesitan las estructuras administrativas de los gobiernos municipales para asignar eficazmente sus recursos? ¿Cuándo comenzaremos a percibir un desarrollo humano sostenido en el país? La discusión apunta a la urgencia de desarrollar capacidades administrativas en los gobiernos municipales, aunque se reconoce que una de las restricciones es el nivel de escolaridad municipal ya que es de la población de donde surgirán los tomadores de decisión. Se intuye de forma muy general que el grado de escolaridad tiene una relación con el manejo de los recursos y las habilidades necesarias en las tareas de gobierno y administración pública. Tenemos dos tareas pendientes a partir de este momento. Los analistas tenemos la tarea urgente de identificar las áreas de oportunidad de los gobiernos locales, en específico de sus capacidades administrativas, entendidas como las habilidades que han desarrollado las estructuras administrativas municipales en torno a las responsabilidades que les han sido asignadas, dentro del marco normativo correspondiente" (p. 103).

\section{Características del Estudio y Contexto de la Investigación}

Con el fin de tener una visión más amplia sobre cada uno de los estudios realizados, en la Tabla No. 1 se muestran sus características. En las dos primeras columnas se presenta el estudio considerado y en la última columna se muestra la base teórica que sustenta la clasificación de cada uno de los rubros considerados. 
Tabla 1. Características de los estudios que integran la investigación

\begin{tabular}{|c|c|c|}
\hline $\begin{array}{l}\text { Inter- } \\
\text { organizacional }\end{array}$ & $\begin{array}{l}\text { Intra- } \\
\text { organizacional }\end{array}$ & Fundamento teórico. \\
\hline $\begin{array}{l}\text { Mixto } \\
\text { (Cualitativo y } \\
\text { Cuantitativo) }\end{array}$ & $\begin{array}{l}\text { Mixto } \\
\text { (Cualitativo y } \\
\text { Cuantitativo) }\end{array}$ & $\begin{array}{l}\text { Se basan en métodos de recolección de datos sin } \\
\text { mediciones numéricas, siendo su propósito el } \\
\text { reconstruir la realidad tal y como la observan los } \\
\text { actores de un sistema social previamente definido. }\end{array}$ \\
\hline $\begin{array}{l}\text { No } \\
\text { experimental }\end{array}$ & $\begin{array}{l}\text { No } \\
\text { experimental }\end{array}$ & $\begin{array}{l}\text { No se manipularon deliberadamente las variables y no } \\
\text { se construyó ninguna situación, sino que se observaron } \\
\text { situaciones ya existentes. }\end{array}$ \\
\hline Transversal & Transversal & $\begin{array}{l}\text { Se recolectaron datos en un solo momento, en un } \\
\text { tiempo único y se proporciona una visión de un evento } \\
\text { o de una situación para describir variables y analizar su } \\
\text { interrelación en un momento dado. }\end{array}$ \\
\hline \multirow[t]{2}{*}{ Exploratorio } & & $\begin{array}{l}\text { Puesto que el propósito fue examinar un tema o } \\
\text { problema de investigación poco estudiado, del cual se } \\
\text { tienen muchas dudas o no se ha abordado antes. }\end{array}$ \\
\hline & Correlacional & $\begin{array}{l}\text { Ya que tiene como propósito evaluar la relación } \\
\text { existente entre dos o más conceptos, categorías o } \\
\text { variables para un contexto en particular. }\end{array}$ \\
\hline
\end{tabular}

Fuente: Elaboración propia con base en Hernández (2010, pp. 5-272).

Para abordar el tema del contexto se debe "tener en cuenta el tipo de sociedad donde se realiza la investigación, la cultura y el medio geográfico" (Mballa, 2017). Sin embargo, no existe una receta para llevarla a cabo, como lo señala Galindo (1998) "decir algo así no tiene gran problema, el punto es desplegar la argumentación sobre esas dimensiones en relación a la actividad reflexiva de segundo nivel. No será lo mismo vivir la experiencia reflexiva en un medio que no promueve las preguntas, que incluso las inhibe o reprime, a vivir en una ecología donde la vivencia estética es el corazón de las relaciones humanas, donde abrir la percepción y enriquecer la conciencia son actividades centrales e indispensables" (p.14).

\section{Elementos del Estudio}

El trabajo de investigación se enfocó en analizar las variaciones que se producen dentro de las estructuras y procesos mismos del sistema, tomando como eje central del estudio el proceso administrativo que se lleva a cabo en departamentos de las administraciones municipales de Tamasopo, Tamuín y Ciudad Valles, S.L.P. Dichos municipios se encuentran en la región denominada Huasteca Norte y comparten características similares en cuanto a aspectos geográficos, climáticos, culturales y gastronómicos.

A continuación, en la Tabla No. 2 se presentan las principales características de los municipios objeto de la presente investigación. 
Tabla 2. Características Generales de los Municipios de la Huasteca Norte

\begin{tabular}{|l|l|l|l|}
\hline & Tamasopo & Tamuín & Ciudad Valles \\
\hline Población & 28,842 habitantes & 37,956 habitantes & 167,713 habitantes \\
\hline Superficie & $1,328.73 \mathrm{Km}^{2}$ & $1,842.66 \mathrm{Km}^{2}$ & $2,396.50 \mathrm{Km}^{2}$ \\
\hline Altura & 360 M.S.N.M. & 20 M.S.N.M. & 70 M.S.N.M. \\
\hline
\end{tabular}

Elaboración propia con base en datos del Instituto Nacional para el Federalismo y Desarrollo Municipal (INAFED).

En lo que se refiere al acceso a la información de las unidades de estudio, las administraciones municipales nos brindaron todo el apoyo para realizar el presente trabajo. La etapa de recolección de información inició en Junio de 2015 y finalizó en Febrero de 2017; a partir de ahí se comenzó con el análisis estadístico de la información y con el desarrollo de conclusiones.

\section{Población objeto de la investigación}

Berenson y Levine (1996, p.3) consideran que "la población es la totalidad de elementos bajo consideración”. En este sentido, para la presente investigación se considera que la aplicación del instrumento fue realizada al total de la población, lo anterior de acuerdo con la información obtenida de los funcionarios municipales quienes dieron constancia que así fue. De este modo, el número de departamentos por municipio y la relación de encuestas aplicadas se muestran en la Tabla No. 3.

Para el estudio se aplicaron 150 encuestas en los tres municipios objeto del estudio, las cuales fueron contestadas por trabajadores de 34 departamentos diferentes; cada aplicación de encuesta tuvo en promedio una duración de 45 minutos.

Tabla 3. Número de Departamentos y Encuestas Aplicadas

\begin{tabular}{|l|l|l|l|l|}
\hline & Tamasopo & Tamuín & $\begin{array}{l}\text { Ciudad } \\
\text { Valles }\end{array}$ & Total \\
\hline Departamentos & 18 & 14 & 2 & 34 Departamentos \\
\hline Encuestas & 31 & 57 & 62 & 150 Encuestas \\
\hline
\end{tabular}

Elaboración propia.

Cabe hacer mención que, en la petición de colaboración a los funcionarios y trabajadores municipales, se estableció un compromiso de anonimato para responder las encuestas, por lo cual éstas no cuentan con el ítem de "nombre"; con esto se asegura el hecho de mantener bajo reserva los nombres de los encuestados.

Para llevar a cabo el proceso de recolección de información se aplicaron 150 encuestas, cada encuesta requirió un promedio de 45 minutos en su aplicación, y éstas se llevaron a cabo en 34 departamentos de los municipios de Tamasopo, Tamuín y Ciudad Valles, S.L.P. 


\section{Definición y operacionalización de variables}

Tomando en consideración que Rodríguez (2003) propone que "el sistema administrativo está relacionado básicamente con la toma de decisiones para planear, organizar, dirigir y controlar el esfuerzo del personal en una organización, el enfoque de sistemas proporciona un marco de referencia integrado para la teoría administrativa y su práctica. La teoría administrativa tradicional utiliza un enfoque basado en un sistema cerrado muy bien estructurado. En contraste, la teoría moderna se ha orientado hacia el enfoque de sistemas abiertos" (p. 174).

Adicionalmente, a lo anterior, Rodríguez (2003) señala que "un enfoque del estudio de los organismos sociales centra su atención en el proceso administrativo fundamental: planeación-organización-integración-dirección y control, que son esenciales si es que la empresa ha de lograr sus objetivos y metas fundamentales. Este proceso administrativo es básico, es necesario para cualquier tipo de empresa (privada, pública, educativa) en la que los recursos humanos, financieros y materiales deben combinarse para lograr ciertos objetivos" (p. 178).

Una vez establecidas las variables a estudiar se procedió a realizar la operacionalización de las mismas. Para llevar a cabo esto, es preciso recordar que para Hernández (2010) una variable "es la propiedad que puede cambiar y cuya transformación es susceptible de medirse" (p. 143). De este modo, se ha diseñado un instrumento que pretende medir las propiedades del proceso administrativo.

La operacionalización de las variables es básica ya que como señala Berenson y Levine (1996) "proporciona un significado a un concepto o variable que puede comunicarse a otros individuos. Es algo que tiene el mismo significado ayer, hoy y mañana para todos los individuos" (p.18). De esta manera, la operacionalización de las variables nos ayudará a procesar información imprescindible para el desarrollo de la investigación.

Podemos decir entonces que el sistema administrativo de una organización se desarrolla en función de sus actividades fundamentales: planeación, organización, integración, dirección y control. Dichas actividades podremos identificarlas como las variables de la presente investigación y son definidas como:

- Planeación. Implica definir los objetivos de la organización, establecer estrategias para lograr dichos objetivos y desarrollar planes para integrar y coordinar actividades de trabajo. (Robbins \& Coulter, 2010, p. 144).

- Organización. Es el proceso para ordenar y distribuir el trabajo, la autoridad y los recursos entre los miembros de una organización, de tal manera que éstos puedan alcanzar las metas de la organización (Stoner, Freeman \& Gilbert Jr., 1996, p.12). 
- Integración de Recursos: Es el conjunto de bienes que es necesarios proveer para que un organismo opere hacia objetivos predeterminados (Rodríguez, 2003, p. 295).

- Dirección: Implica mandar, influir y motivar a los empleados para que realicen tareas esenciales. La dirección llega al fondo de las relaciones de los gerentes con cada una de las personas que trabajan con ellos (Stoner, Freeman \& Gilbert Jr., 1996, p.13).

- Control: Proceso para asegurar que las actividades reales se ajustan a las actividades planificadas (Stoner, Freeman \& Gilbert Jr., 1996, p.13).

Una vez que se identificaron las variables se elaboró una encuesta, la cual como señalan Berenson y Levine (1996) "es un método para obtener datos, en el cual no se ejerce ningún control sobre el comportamiento de la gente encuestada. Simplemente se formulan preguntas respecto a sus opiniones, actitudes, comportamiento y otras características. Después sus respuestas se editan, codifican y tabulan para su análisis" (p. 14). El objetivo de dicha encuesta fue servir como instrumento de medición, el cual consta de varias preguntas agrupadas en los cinco elementos que integran el proceso administrativo y cuyo fin es medir la implementación de los elementos del proceso administrativo.

Cabe hacer mención que las categorías de respuesta de la encuesta fueron a través de una escala de Likert, utilizando las siguientes categorías: a) Nunca, b) A veces, c) Frecuentemente, d) Muy Frecuentemente, y d) Siempre.

\section{Tratamiento estadístico}

Una vez recolectados los datos, se realizó el tratamiento estadístico de los mismos por medio de las siguientes acciones:

1. Se diseñó la base de datos, estableciéndose de manera tal que las características correspondieran a los aspectos que se pretendían medir. Para lo anterior se empleó el software Statistical Package for the Social Sciences SPSS versión 19 (en lo sucesivo SPSS).

2. Se realizó la captura de la información de las 150 encuestas realizadas correspondientes al personal de los municipios objeto del estudio: Tamasopo, Tamuín y Ciudad Valles, S.L.P., verificando de manera aleatoria si los datos capturados eran correctos.

3. Se realizó un proceso de datos ausentes, que de acuerdo con Hair et al. (2005, p. 39) "es cualquier evento sistemático externo al encuestado tales como errores en la introducción de los datos, problemas de recolección o bien encuestas incompletas por la negativa de contestar por parte del entrevistado". Las acciones que se realizaron para identificar y remediar el problema fueron las siguientes:

$\checkmark$ Ubicar los casos de datos faltantes identificando el número de ítem y el número de encuesta. 
Se revisó que la captura de las respuestas de la encuesta correspondiente fuera correcta y se alimentó el dato que no se había considerado; en la Tabla No. 3 se puede observar el resumen general del procesamiento de los casos.

En el caso de las encuestas cuyas respuestas eran incompletas se procedió a no considerarlas (no se detectaron encuestas con esta característica). Una vez realizado lo anteriormente expuesto, se determinó que no existieron datos ausentes tal y como lo muestra la Tabla No. 4 obtenida del Software SPSS.

Tabla 1 Resumen del procesamiento de los casos

\begin{tabular}{|c|c|c|c|}
\hline & & Número & Porcentaje $\%$ \\
\hline \multirow{3}{*}{ Casos } & Válidos & 150 & 100.0 \\
\hline & Excluidos $^{a}$ & 0 & 0.0 \\
\hline & Total & 150 & 100.0 \\
\hline
\end{tabular}

Fuente: Elaboración Propia.

4. Se obtuvieron las estadísticas descriptivas básicas, lo que permitió establecer cómo estaba conformada la población de los departamentos encuestados y cuáles eran las características principales de los encuestados.

5. Se obtuvo la confiabilidad del cuestionario utilizado, debido a que, de acuerdo con Hernández, S. et al. (2010), "la confiabilidad de un instrumento de medición se refiere al grado en que su aplicación repetida al mismo sujeto u objeto, produce resultados iguales" (p.346). A continuación, en la Tabla No. 5 , se puede observar el grado de confiabilidad del instrumento.

Tabla 2. Confiabilidad del instrumento de captación de información

\begin{tabular}{|l|l|l|}
\hline Variable & Número de ítems & Alfa de Cronbach \\
\hline Planeación & 11 & 0.932 \\
\hline Organización & 5 & 0.928 \\
\hline Integración & 11 & 0.922 \\
\hline Dirección & 11 & 0.945 \\
\hline Control & 10 & 0.834 \\
\hline Total & 48 & 0.975 \\
\hline
\end{tabular}

Elaboración propia.

Para establecer la confiabilidad del instrumento se utilizó la prueba estadística del coeficiente alfa de Cronbach integrada al software SPSS, la cual determina la proporción en que los ítems de una prueba miden una misma característica en común. De acuerdo con Hair et al., (2005) "el acuerdo general sobre el límite inferior para el alfa de Cronbach es de 0.70, aunque puede bajar a 0.60 en la investigación exploratoria”. A su vez, Pardo y Ruiz (2005, p. 598) señalan que los valores por encima de 0.80 se suelen considerar meritorios y los valores por encima de 0.90 excelentes" (p. 105). En el caso de nuestro estudio los resultados de confiabilidad se encontraron en un rango de $0.83 \mathrm{y}$ 
hasta 0.97 , lo cual indica que los resultados están por encima de ser meritorios tal y como se muestra en la Tabla No. 5.

\section{Discusión de Resultados y Reflexiones Finales}

En el presente apartado se presentarán los resultados de las tablas de contingencias obtenidas de la base de datos de la encuesta aplicada a trabajadores de las administraciones públicas municipales de los municipios de Tamasopo, Tamuín y Ciudad Valles, S.L.P.

En la Tabla No. 6 se puede observar que en el municipio de Tamasopo se visitaron 18 departamentos en los cuales se aplicaron 31 encuestas; en el municipio de Tamuín se visitaron 14 departamentos y se aplicaron 57 encuestas y finalmente en el municipio de Ciudad Valles se visitaron 2 departamentos y se aplicaron 62 encuestas.

Tabla 3. Departamentos por Municipio

\begin{tabular}{|l|l|l|l|l|}
\hline Municipio & Tamasopo & Tamuín & Ciudad Valles & Total \\
\hline Departamentos & 18 & 14 & 2 & 34 \\
\hline Encuestas & 31 & 57 & 62 & 150 \\
\hline
\end{tabular}

Fuente: Elaboración propia.

Tabla 4. Nombramientos de los Trabajadores por Municipio

\begin{tabular}{|l|l|l|l|l|}
\hline \multicolumn{3}{|c|}{ Nombramiento } & Total \\
\hline \multirow{4}{*}{ Municipio } & Jefe de Departamento & Operativo & \\
\cline { 2 - 6 } & Tamasopo & 15 & 16 & 31 \\
\cline { 2 - 6 } & Tamuín & 11 & 46 & 57 \\
\cline { 2 - 6 } & Ciudad Valles & 24 & 38 & 62 \\
\hline Total & 50 & 100 & 150 \\
\hline
\end{tabular}

Fuente: Elaboración propia.

Continuando con el análisis en la Tabla No. 7 se puede observar que en el municipio de Tamasopo se encuestaron a 15 jefes de departamento, así como a 16 trabajadores operativos, para un total de 31 encuestas en ese municipio. En lo que respecta al municipio de Tamuín se realizaron 11 encuestas a jefes de departamento y 46 a trabajadores operativos para un total de 57 encuestas, mientras que en el municipio de Ciudad Valles se encuestaron a 24 jefes de departamento, así como a 38 trabajadores operativos para un total de 62 encuestas aplicadas.

Una variable importante para medir el grado de profesionalización de las organizaciones es el análisis de la escolaridad de sus trabajadores. Por esta razón en la Tabla No. 8 podemos identificar que en el municipio de Tamasopo 7 personas cuentan con estudios técnicos de bachillerato, licenciatura o posgrado, mientras que sólo 4 tienen estudios de secundaria; no existen registros de personal con primaria. En lo que respecta al municipio de Tamuín, 8 de las personas encuestadas cuentan con estudios de Técnico o un nivel posterior mientras que ocho cuentan con estudios de secundaria y tres con 
estudios de primaria. Finalmente, en el municipio de Ciudad Valles 13 personas de las encuestadas cuentan con estudios de técnico o un nivel posterior, tan sólo una persona con estudios de secundaria y ninguna persona con estudios de primaria. Lo anterior muestra en los tres municipios una preocupación evidente por contar con personal especializado para realizar sus labores.

Tabla 5. Escolaridad de la Población Encuestada

\begin{tabular}{|c|c|c|c|c|c|c|}
\hline \multirow[b]{2}{*}{ Municipio } & \multicolumn{5}{|l|}{ Escolaridad } & \multirow[b]{2}{*}{ Total } \\
\hline & PrimariaSe & Té & $\mathrm{Bac}$ & Lic & $\mathrm{aPo}$ & \\
\hline Tamasopo & \begin{tabular}{l|l}
0 & 4
\end{tabular} & 7 & 10 & 10 & 0 & 31 \\
\hline Tamuín & 3 & 8 & 12 & 25 & 1 & 57 \\
\hline Ciudad Valles & 0 & 10 & 13 & 36 & 2 & 62 \\
\hline Total & 13 & 25 & 35 & 71 & 3 & 150 \\
\hline
\end{tabular}

Fuente: Elaboración propia.

\section{Análisis de Varianza (ANOVA)}

Para Berenson y Levine (1996) en ocasiones es "necesario evaluar las diferencias entre los parámetros de varios $(c)$ grupos. Cuando las mediciones resultantes en los $(c)$ son continuas y se cumplen ciertas suposiciones, se puede emplear una metodología conocida como análisis de varianza (o ANOVA; por su nombre: ANalysis Of VAriance) para comparar las medias de los grupos. En un cierto sentido, el término "análisis de varianza" parece no ser el correcto, pues el objetivo consiste en analizar diferencias entre las medias de los grupos. Sin embargo, a través del análisis de la variación de los datos, tanto entre los (c) grupos como dentro de ellos, seremos capaces de llegar a conclusiones acerca de posibles diferencias en las medidas en las medias de los grupos. En el ANOVA subdividimos la variación total de las mediciones resultantes en lo que se puede atribuir a diferencias entre los (c) grupos y lo que se debe al azar o que se puede atribuir a una variación inherente dentro de los (c) grupos. La variación dentro de los grupos se considera error experimental, mientras que la variación entre grupos se atribuye a efectos de tratamiento (p. 528).

Para llevar a cabo el análisis ANOVA fue utilizado el paquete estadístico SPPP versión 19 y las tablas tal como fueron desplegadas por el programa (las mismas pueden ser consultadas en el anexo del presente trabajo). Las tablas presentadas en éste apartado muestran las variables relacionadas, el nivel de significancia considerado $(\alpha<0.05)$; el número de actividades realizadas de las variables de las funciones administrativas $(1=$ nunca; $2=$ a veces; 3 = frecuentemente; $4=$ muy frecuentemente $; 5=$ siempre); así como una breve interpretación de los resultados. Dichas respuestas fueron analizadas en función del nivel de escolaridad de los encuestados ( 1 = Primaria incompleta; $2=$ Primaria; $3=$ Secundaria; 4 = Técnico; $5=$ Bachillerato; $6=$ Licenciatura y $7=$ Posgrado). 


\section{Análisis de Varianza (Anovas) aplicado a la variable Planeación}

A continuación, se presentará la información obtenida al emplear el estadístico ANOVA aplicado a variables de planeación y comparándola con la variable de escolaridad, esto con el fin de determinar el grado de significancia que el grado de estudios puede tener en la sistematización de los procesos administrativos de los municipios de la huasteca norte.

De acuerdo con la escala de nuestro instrumento, podemos observar en la Tabla No. 9 que en el municipio de Tamasopo los jefes de departamento participan muy frecuentemente, y los jefes de Tamuín y Ciudad Valles frecuentemente, lo que nos indica que las medias de estos tres grupos no son iguales. Cabe mencionar que el nivel de significancia en los tres casos es de 0.00 , el cual es menor al Alfa que manejamos de 0.05. Con este resultado la hipótesis de que las medias son iguales se rechaza. Aunado a lo anterior, se puede observar que el grado de escolaridad en para el caso de los jefes se encuentra en el municipio de Tamasopo con una media de 4.47 la cual corresponde a un nivel técnico, mientras que para el caso de Tamuín y Ciudad Valles se ubica por encima del número 5, lo que corresponde a un nivel de Bachillerato en promedio.

Tabla 6. ANOVA: Media de Participación en Diseño de Manuales de Procedimientos de los Jefes de los Municipios de Tamasopo, Tamuín y Ciudad Valles, S.L.P.

\begin{tabular}{|l|l|l|l|}
\hline Grupos & $\begin{array}{l}\text { Media de Participación } \\
\text { en la Planeación }\end{array}$ & $\begin{array}{l}\text { Media } \\
\text { Escolaridad }\end{array}$ & Significancia \\
\hline Tamasopo & 4.07 & 4.47 & 0.00 \\
\hline Tamuín & 3.91 & 5.55 & 0.00 \\
\hline Ciudad Valles & 3.29 & 5.58 & 0.00 \\
\hline
\end{tabular}

Fuente: Elaboración propia.

De igual forma, en la Tabla No. 9 se puede apreciar que a medida que la preparación profesional aumenta en el caso de los Jefes, su percepción de participación en las actividades de planeación disminuye; tal es el caso de Ciudad Valles, en dónde la media de preparación se ubica en 5.58 que corresponde a nivel Bachilleratoy, sin embargo, la percepción de participación se ubica en la categoría "frecuente".

En lo que respecta a la participación de los trabajadores operativos en la planeación, en la Tabla No. 10 se puede observar que las medias de participación en el diseño de manuales de procedimientos se ubican entre un 2.00 y un 2.33 , lo cual corresponde a la categoría "a veces", contrastando esto con la opinión de los jefes presentada en la Tabla No. 9, tabla donde éstos últimos señalaban que la participación en la organización corresponde a la categoría "frecuentemente".

Cabe hacer mención de que la media de participación en la planeación correspondiente a los trabajadores operativos de los municipios de Tamasopo se ubica en 2.33, Tamuín 2.00 y Ciudad Valles 2.00. En todos los casos 
corresponde a la categoría "a veces", con lo cual podemos concluir que las medias de estos tres grupos son iguales. Se pudo observar igualmente que el grado de escolaridad en para el caso de los operativos se encuentra en el municipio de Tamasopo con una media de 4.74 la cual corresponde a un nivel técnico, mientras que para el caso de Tamuín y Ciudad Valles se ubica por encima del número 5 lo que corresponde a un nivel de Bachillerato en promedio.

Tabla 7. ANOVA: Media de Participación en Diseño de Manuales de Procedimientos de los Trabajadores de los Municipios de Tamasopo, Tamuín y Ciudad Valles, S.L.P.

\begin{tabular}{|l|l|l|l|}
\hline Grupos & $\begin{array}{l}\text { Media de Participación } \\
\text { en la Planeación }\end{array}$ & $\begin{array}{l}\text { Media } \\
\text { Escolaridad }\end{array}$ & Significancia \\
\hline Tamasopo & 2.33 & 4.74 & 0.00 \\
\hline Tamuín & 2.00 & 5.19 & 0.00 \\
\hline Ciudad Valles & 2.00 & 5.37 & 0.00 \\
\hline
\end{tabular}

Fuente: Elaboración propia.

\section{Análisis de Varianza (Anovas) aplicado a la variable Organización}

A continuación, se presentará la información obtenida al emplear el estadístico ANOVA aplicado a variables de organización y comparándola con la variable de escolaridad con el fin de determinar el grado de significancia que el grado de estudios puede tener en la sistematización de los procesos administrativos de los municipios de la huasteca norte.

Tabla 8. ANOVA: Media de la Percepción de los Jefes de los Municipios de Tamasopo, Tamuín y Ciudad Valles, S.L.P. con respecto a si su Dependencia Cuenta con Manuales

\begin{tabular}{|l|l|l|l|}
\hline Grupos & $\begin{array}{l}\text { Media de Participación } \\
\text { en la Organización }\end{array}$ & $\begin{array}{l}\text { Media } \\
\text { Escolaridad }\end{array}$ & Significancia \\
\hline Tamasopo & 3.80 & 4.47 & 0.00 \\
\hline Tamuín & 4.36 & 5.55 & 0.00 \\
\hline Ciudad Valles & 4.75 & 5.58 & 0.00 \\
\hline
\end{tabular}

Fuente: Elaboración propia.

De acuerdo con la escala de nuestro instrumento podemos observar en la Tabla No. 11 que en el municipio de Tamasopo los jefes de departamento perciben que la dependencia cuenta con manuales "frecuentemente" al ubicarse su media en 3.80, mientras que los jefes de Tamuín y Ciudad Valles perciben que es "muy frecuentemente" al ubicarse en 4.36 y 4.75 respectivamente, lo que nos indica que las medias de estos tres grupos no son iguales.

En lo que respecta a la percepción de los trabajadores operativos en la variable organización en la Tabla No. 12, se puede observar que las medias de percepción con respecto a si su dependencia cuenta con manuales se ubican en 2.31 para el municipio de Tamasopo que corresponde a la categoría "a veces", mientras que para los municipios de Tamasopo y Ciudad Valles se ubica en 3.13 y 3.79 respectivamente correspondiendo a la categoría 
"frecuentemente" A partir de estos resultados podemos concluir que las medias de estos tres grupos no son iguales.

Tabla 9. ANOVA: Media de Percepción de Trabajadores de los Municipios de Tamasopo, Tamuín y Ciudad Valles, S.L.P. con respecto a si su Dependencia Cuenta con Manuales

\begin{tabular}{|l|l|l|l|}
\hline Grupos & $\begin{array}{l}\text { Media de Participación } \\
\text { en la Organización }\end{array}$ & $\begin{array}{l}\text { Media } \\
\text { Escolaridad }\end{array}$ & Significancia \\
\hline Tamasopo & 3.13 & 4.74 & 0.00 \\
\hline Tamuín & 2.31 & 5.19 & 0.00 \\
\hline Ciudad Valles & 3.79 & 5.37 & 0.00 \\
\hline
\end{tabular}

Fuente: Elaboración propia.

\section{Análisis de Varianza (Anova) aplicado a la variable Integración}

A continuación, se presentará la información obtenida al emplear el estadístico ANOVA aplicado a variables del paso del proceso administrativo denominado Integración tomando como referencia la variable de escolaridad con el fin de determinar el grado de significancia que el grado de estudios puede tener en la sistematización de los procesos administrativos de los municipios de la huasteca norte.

Tabla 10. ANOVA: Media de la Percepción de los Jefes de los Municipios de Tamasopo, Tamuín y Ciudad Valles, S.L.P. con respecto al suministro de los recursos tecnológicos y materiales de tal forma que permitan llevar a cabo su trabajo de manera eficiente

\begin{tabular}{|l|l|l|l|}
\hline Grupos & $\begin{array}{l}\text { Media de Participación } \\
\text { en la Integración }\end{array}$ & $\begin{array}{l}\text { Media } \\
\text { Escolaridad }\end{array}$ & Significancia \\
\hline Tamasopo & 3.13 & 4.47 & 0.00 \\
\hline Tamuín & 3.64 & 5.55 & 0.00 \\
\hline Ciudad Valles & 4.33 & 5.58 & 0.00 \\
\hline
\end{tabular}

Fuente: Elaboración propia.

De acuerdo con la escala de nuestro instrumento podemos observar en la Tabla No. 13 que en los municipios de Tamasopo y Tamuín los jefes de departamento perciben que existe un suministro frecuente de los recursos tecnológicos y materiales para llevar a cabo su trabajo de manera eficiente al ubicarse sus medias en 3.13 y 3.64 respectivamente, mientras que en el municipio de Ciudad Valles los jefes de departamento cuentan con una media de 4.33 lo cual indica que su percepción se encuentra en la categoría "muy frecuentemente" También se puede observar que a medida que la escolaridad aumenta también aumenta la percepción de que las actividades de integración mejoran en el proceso administrativo de sus administraciones.

Por su parte, en cuanto a la percepción de los trabajadores operativos en la variable Integración, en la Tabla No. 14 se puede observar que las medias de los trabajadores operativos se ubican en la categoría "a veces"al registrar una media en Tamasopo de 2.80 y en Tamuín de 2.75 , mientras que para el caso de Ciudad Valles la media se ubica en 3.63, indicando que la percepción se ubica en la categoría "frecuentemente" con respecto al suministro de los 
recursos tecnológicos y materiales para llevar a cabo su trabajo de manera eficiente.

Tabla 11. ANOVA: Media de Percepción de Trabajadores de los Municipios de Tamasopo, Tamuín y Ciudad Valles, S.L.P. con respecto al suministro de los recursos tecnológicos y materiales de tal forma que permitan llevar a cabo su trabajo de manera eficiente

\begin{tabular}{|l|l|l|l|}
\hline Grupos & $\begin{array}{l}\text { Media de Participación } \\
\text { en la Integración }\end{array}$ & $\begin{array}{l}\text { Media } \\
\text { Escolaridad }\end{array}$ & Significancia \\
\hline Tamasopo & 2.80 & 4.74 & 0.00 \\
\hline Tamuín & 2.75 & 5.19 & 0.00 \\
\hline Ciudad Valles & 3.63 & 5.37 & 0.00 \\
\hline
\end{tabular}

Fuente: Elaboración propia.

Cabe mencionar que la media de percepción de los trabajadores operativos con respecto al suministro de los recursos tecnológicos y materiales de tal forma que permitan llevar a cabo su trabajo de manera eficiente en los municipios de Tamasopo se ubica en 2.80 que corresponde a la categoría "a veces", en Tamuín 2.75 que corresponde a la categoría "a veces" y en Ciudad Valles 3.63 que corresponde a la categoría "frecuentemente", con lo cual podemos concluir que las medias de estos tres grupos no son iguales.

\section{Análisis de Varianza (ANOVA) aplicado a la variable Dirección}

A continuación, se presentará la información obtenida al emplear el estadístico ANOVA aplicado a variables del paso del proceso administrativo denominado Dirección y tomando como referencia la variable de escolaridad con el fin de determinar el grado de significancia que el grado de estudios puede tener en la sistematización de los procesos administrativos de los municipios de la huasteca norte.

De acuerdo con la escala de nuestro instrumento podemos observar en la Tabla No. 15 que en los municipios de Tamasopo y Tamuín los jefes de departamento perciben de manera frecuente que existe una comunicación de estrategias por parte de la Dependencia para el logro de los objetivos al ubicarse sus medias en 3.13 y 3.82 respectivamente, mientras que en el municipio de Ciudad Valles los jefes de departamento cuentan con una media de 4.46, lo cual indica que su percepción se encuentra en la categoría "muy frecuentemente". De igual manera se puede observar que a medida que la escolaridad aumenta también aumenta la percepción de que las actividades de dirección mejoran en el proceso administrativo de sus administraciones, dado que en el municipio de Tamasopo la media de escolaridad se ubica en la categoría de Técnico, mientras que en Tamuín y Ciudad Valles aumenta a la categoría de Bachillerato. 
Tabla 12. Media de la Percepción de los Jefes de los Municipios de Tamasopo, Tamuín y Ciudad Valles, S.L.P. con respecto a la Comunicación de Estrategias por parte de la

Dependencia para el Logro de los Objetivos

\begin{tabular}{|l|l|l|l|}
\hline Grupos & $\begin{array}{l}\text { Media de Participación } \\
\text { en la Dirección }\end{array}$ & $\begin{array}{l}\text { Media } \\
\text { Escolaridad }\end{array}$ & Significancia \\
\hline Tamasopo & 3.13 & 4.47 & 0.00 \\
\hline Tamuín & 3.82 & 5.55 & 0.00 \\
\hline Ciudad Valles & 4.46 & 5.58 & 0.00 \\
\hline
\end{tabular}

Fuente: Elaboración propia.

Por su parte, en cuanto a la percepción de los trabajadores operativos en la variable Dirección en la Tabla No. 16, se puede observar que las medias de los trabajadores operativos se ubican en la categoría "a veces" al registrar una media en Tamasopo de 2.57 y en Tamuín de 2.06, mientras que para el caso de Ciudad Valles la media se ubica en 3.32, indicando indica que la percepción se ubica en la categoría "frecuentemente" con respecto a la comunicación de estrategias por parte de la Dependencia para el logro de los objetivos, con lo cual podemos concluir que las medias de estos tres grupos no son iguales.

Tabla 13. ANOVA: Media de Percepción de Trabajadores de los Municipios de

Tamasopo, Tamuín y Ciudad Valles, S.L.P. con respecto a la Comunicación de Estrategias por parte de la Dependencia para el Logro de los Objetivos

\begin{tabular}{|l|l|l|l|}
\hline Grupos & $\begin{array}{l}\text { Media de Participación } \\
\text { en la Dirección }\end{array}$ & $\begin{array}{l}\text { Media } \\
\text { Escolaridad }\end{array}$ & Significancia \\
\hline Tamasopo & 2.57 & 4.74 & 0.00 \\
\hline Tamuín & 2.06 & 5.19 & 0.00 \\
\hline Ciudad Valles & 3.32 & 5.37 & 0.00 \\
\hline
\end{tabular}

Fuente: Elaboración propia.

\section{Análisis de Varianza (ANOVA) aplicado a la variable Control}

A continuación, se presentará la información obtenida al emplear el estadístico ANOVA aplicado a variables del paso del proceso administrativo denominado Control y tomando como referencia la variable de escolaridad con el fin de determinar el grado de significancia que el grado de estudios puede tener en la sistematización de los procesos administrativos de los municipios de la huasteca norte.

De acuerdo con la escala de nuestro instrumento podemos observar en la Tabla No. 17 que en el municipio de Tamasopo la media de los Jefes con respecto a la percepción de las acciones para eliminar las causas de fallas y errores para evitar su repetición se ubica en 3.40 que corresponde a la categoría "frecuentemente", mientras que en los municipios de Tamuín y Ciudad Valles se ubican en 4.09 y 4.17 respectivamente y corresponden a la categoría "muy frecuentemente", lo cual nos indica que las medias de estos tres grupos no son iguales. 
Tabla 14. ANOVA: Media de la Percepción de los Jefes de los Municipios de Tamasopo, Tamuín y Ciudad Valles, S.L.P. con respecto a las Acciones para Eliminar las Causas de Fallas y Errores para Evitar su Repetición

\begin{tabular}{|l|l|l|l|}
\hline Grupos & $\begin{array}{l}\text { Media de Participación } \\
\text { en la Control }\end{array}$ & $\begin{array}{l}\text { Media } \\
\text { Escolaridad }\end{array}$ & Significancia \\
\hline Tamasopo & 3.40 & 4.47 & 0.00 \\
\hline Tamuín & 4.09 & 5.55 & 0.00 \\
\hline Ciudad Valles & 4.17 & 5.58 & 0.00 \\
\hline
\end{tabular}

Fuente: Elaboración propia.

Además, se puede observar que a medida que la escolaridad aumenta también aumenta la percepción de que las actividades de control mejoran en el proceso administrativo de sus administraciones, dado que en el municipio de Tamasopo la media de escolaridad se ubica en 4.47 y corresponde a la categoría de Técnico, mientras que en Tamuín y Ciudad Valles aumenta a 5.55 y 5.58 respectivamente las cuales corresponden a la categoría de Bachillerato.

Tabla 15. ANOVA: Media de Percepción de Trabajadores de los Municipios de Tamasopo, Tamuín y Ciudad Valles, S.L.P. con respecto a las Acciones para Eliminar las Causas de Fallas y Errores para Evitar su Repetición

\begin{tabular}{|l|l|l|l|}
\hline Grupos & $\begin{array}{l}\text { Media de Participación } \\
\text { en la Control }\end{array}$ & $\begin{array}{l}\text { Media } \\
\text { Escolaridad }\end{array}$ & Significancia \\
\hline Tamasopo & 2.67 & 4.74 & 0.00 \\
\hline Tamuín & 2.56 & 5.19 & 0.00 \\
\hline Ciudad Valles & 3.76 & 5.37 & 0.00 \\
\hline
\end{tabular}

Fuente: Elaboración propia.

Por su parte, en cuanto a la percepción de los trabajadores operativos en la variable Control en la Tabla No. 18 se puede observar que las medias de los trabajadores operativos se ubican en la categoría "a veces" al registrar una media en Tamasopo de 2.67 y en Tamuín de 2.56, mientras que para el caso de Ciudad Valles la media se ubica en 3.76 lo cual indica que la percepción se ubica en la categoría "frecuentemente" con respecto a las acciones para eliminar las causas de fallas y errores en la Dependencia y evitar su repetición, resultados a partir de los cuales podemos concluir que las medias de estos tres grupos no son iguales.

\section{Consideraciones Finales}

La Administración, al igual que muchas Ciencias y Disciplinas, ha evolucionado buscando las mejores respuestas para que la Sociedad viva de manera más adecuada al contexto y buscando eficientar los recursos a su alcance. En el caso de la Administración, las primeras teorías se remontan al estudio clásico de la disciplina propuesto por Frederick Winslow Taylor con su trabajo sobre la Administración Científica, así como a Henri Fayol con su obra Administración Industrial y General, estudios gracias a los cuales ambos autores son considerados los padres de la Administración. 
Posterior a Taylor y Fayol la administración ha sido estudiada desde diferentes perspectivas, entre las cuales podemos mencionar las teorías de la burocracia, las teorías de las relaciones humanas, las escuelas del comportamiento, las escuelas del análisis del poder y el discurso organizacional, por mencionar las más importantes. Cada una de estas escuelas ha brindado nuevas herramientas para eficientar el trabajo de los administradores y han sido un paso importante en la evolución del pensamiento administrativo.

Hoy en día la problemática financiera y administrativa ha invadido también el caso de la administración pública y en el caso de sus dependencias éstas deben enfrentar un contexto complicado en un ámbito globalizado, en el cual se vuelven necesarias la cooperación y el trabajo coordinado con los diferentes agentes políticos y civiles, con el fin de brindar una respuesta más eficaz a las necesidades sociales.

Sumado a las necesidades colectivas hoy encontramos a una sociedad más ávida de información por ello el gobierno ha tenido que implementar nuevos mecanismos de comunicación con la ciudadanía tales como el gobierno electrónico, con el fin de brindar mayor transparencia y rendición de cuentas ante una sociedad cada día más interesada en los temas de la administración pública.

Por tal motivo el tema de la administración pública y la eficiencia en sus procesos es un tema de mucho interés hoy en día. La sociedad cada vez cuenta con mayor acceso a la información y ello representa un mayor cuestionamiento de las decisiones, decisiones que son tomadas en diferentes ámbitos de la administración pública, tal y como lo señala Castellanos (2014) quién expresa que "en el ámbito del gobierno y la administración pública es muy fácil identificar tres procesos de tomas de decisiones: en el ámbito legislativo, en el ámbito jurisdiccional judicial, y desde luego, el proceso administrativo público" (p.72).

De este modo el presente trabajo en primer término presentó una herramienta diseñada como propuesta metodológica para ayudar a las administraciones municipales a mejorar sus procesos administrativos, atendiendo cada una de las fases que lo integran (Planeación, Organización, Integración, Dirección y Control) con el fin de identificar en que parte del proceso se están produciendo fallas que puedan ser detectadas y aplicar medidas correctivas con el fin de llevar a cabo procesos de mejora continua.

En segundo término el documento presentó un estudio en donde dicha metodología se pone en práctica y en la cual se pudo documentar que para el caso de los municipios de Tamasopo, Tamuín y Ciudad Valles en el Estado de San Luis Potosí, resulta que se detecta una falta de sistematización en los procesos administrativos, aunado a una opinión diferenciada entre la percepción que tienen los Jefes de Departamento en cuanto a la aplicación del 
Proceso Administrativo y la opinión que tienen los trabajadores operativos con respecto a las fases de Planeación, Organización, Integración, Dirección y Control.

Lo anteriormente expuesto nos permite entonces afirmar que la presente investigación sirve de base para la realización de futuras investigaciones en torno al tema de la eficiencia en los procesos administrativos de las administraciones públicas en el entendido de que día a día la población es más crítica y demanda una mejor calidad de servicios, así como una mejor rendición de cuentas a cambio de las contribuciones que eroga durante cada ejercicio fiscal.

\section{References:}

1. Berenson Mark, L. \& David Levine, M. (1996). "Estadística Básica en Administración", Editorial Pearson Prentice Hall, Segunda Edición en español, México.

2. Castellanos, Hernández, \& Eduardo de Jesús (2014). "El Proceso Administrativo Público. Fundamento Constitucional", en Revista de Administración Pública, Volumen XLIX 2.

3. Fayol Henry (1987). "Administración Industrial y General", Decimocuarta Edición, Librería "El Ateneo" Editorial, Argentina.

4. Galindo Cáceres Luis Jesús (1998). "Técnicas de Investigación”; En Sociedad, Cultura y Comunicación, Editorial Pearson, Primera Edición, México.

5. Hair Jr. Joseph, F., Anderson Rolph, Tatham Ronald, L., \& Black William, C. (2005). "Análisis Multivariante", Editorial Pearson Prentice Hall, España.

6. Hernández, S., Roberto, Carlos Fernández, C., \& Pilar Baptista, L. (2010). "Metodología de la Investigación", Editorial McGrawHill, Quinta Edición, Chile.

7. Martínez Andrade \& Juan Carlos (2014). "Gobierno, administración y desarrollo humano en los gobiernos locales", en Revista de Administración Pública, México, Volumen XLIX, No. 2.

8. Mballa Louis Valentin (2017). "Los avatares del interés de nido en términos de poder en la formulación de las políticas públicas". Rev. Brasileira de Políticas Públicas, Brasília, v. 7, no 3, 2017 p. 799-817.

9. Mballa Louis Valentin (2018). "Vers des fondements de la rationalité complexe pour l'analyse de la politique publique : les avatars du processus décisionnel": Rev. Ciencia política, 13(26), 261-286.

10. Merino Mauricio (2004). "Los Gobiernos Municipales de México: El Problema del Diseño Institucional", Documento de Trabajo No. 145, Centro de Investigación y Docencia Económicas (CIDE), México. 
11. Pardo Merino Antonio \& Miguel Ángel Ruiz Díaz (2005). “Análisis de datos con SPSS 13 Base", Editorial McGraw-Hill, México.

12. Peters Guy (2005). "Gobernanza y Burocracia: ¿Nuevas formas de democracia o nuevas formas de control?"; Foro Internacional, 182, XLV, Colmex, México.

13. Robbins Stepehen, P. \& Mary Coulter (2010). "Administración", Décima Edición, Pearson Educación, México.

14. Rodríguez V. Joaquín (2003). "Introducción a la Administración con el Enfoque de Sistemas", Editorial Thomson, Cuarta Edición, México.

15. Stoner, J., Freeman, R., \& Gilbert, D. (1996). “Administración”, Sexta Edición, Editorial Pearson Prentice Hall, México.

16. Taylor Frederick, W. (1987). "Principios de la Administración Científica", Décima Edición, Librería "El Ateneo" Editorial, Argentina.

17. Páginas

de

Internet http://www.inafed.gob.mx/work/enciclopedia/EMM24sanluispotosi/i ndex.html Instituto Nacional para el Federalismo y Desarrollo Municipal 